\title{
Spectrally resolved four-wave mixing in semiconductors: Influence of inhomogeneous broadening
}

\author{
Erland, J.; Pantke, K.-H.; Mizeikis, V.; Vadim, Lyssenko; Hvam, Jørn Märcher
}

Published in:

Physical Review B

Link to article, DOI:

10.1103/PhysRevB.50.15047

Publication date:

1994

Document Version

Publisher's PDF, also known as Version of record

Link back to DTU Orbit

Citation (APA):

Erland, J., Pantke, K-H., Mizeikis, V., Vadim, L., \& Hvam, J. M. (1994). Spectrally resolved four-wave mixing in semiconductors: Influence of inhomogeneous broadening. Physical Review B, 50(20), 15047-15055.

https://doi.org/10.1103/PhysRevB.50.15047

\section{General rights}

Copyright and moral rights for the publications made accessible in the public portal are retained by the authors and/or other copyright owners and it is a condition of accessing publications that users recognise and abide by the legal requirements associated with these rights.

- Users may download and print one copy of any publication from the public portal for the purpose of private study or research.

- You may not further distribute the material or use it for any profit-making activity or commercial gain

- You may freely distribute the URL identifying the publication in the public portal 


\title{
Spectrally resolved four-wave mixing in semiconductors: Influence of inhomogeneous broadening
}

\author{
J. Erland, K.-H. Pantke, and V. Mizeikis* \\ Fysisk Institut, Odense Universitet, Campusvej 55, DK-5230 Odense M, Denmark \\ V. G. Lyssenko ${ }^{\dagger}$ and J. M. Hvam \\ Mikroelektronik Centret, The Technical University of Denmark, DK-2800 Lyngby, Denmark
} (Received 31 May 1994)

\begin{abstract}
We study the influence of inhomogeneous broadening on results obtained from spectrally resolved transient four-wave mixing. In particlar, we study the case where more resonances are coherently excited, leading to polarization interference or quantum beats, depending on the microscopic nature of the resonances. To demonstrate the ideas, we present experimental results from bulk CdSe and from GaAs multiple quantum wells.
\end{abstract}

\section{INTRODUCTION}

Current issues in the exploration of light-matter interactions are coherence and quantum interference. ${ }^{1}$ With ultrafast nonlinear spectroscopy such as transient four-wave mixing (TFWM), the coherence of the optical excitations in semiconductors and semiconductor structures is studied with femtosecond time resolution, resolving typical dephasing times of excitonic resonances. In these experiments, the laser beam with ultrashort pulses is split into two beams incident on the sample in the directions $\mathbf{k}_{1}$ and $\mathbf{k}_{2}$, and the nonlinear TFWM signal is emitted in the background-free direction $2 \mathbf{k}_{2}-\mathbf{k}_{1}$, and usually detected time integrated as a function of the delay $\tau$. These correlation traces will, for delays much larger than the pulse length, and for systems that can reasonably well be described by a two-level system, ${ }^{2}$ show an exponential decay $I_{\mathrm{FWHM}}(\tau) \propto I(0) e^{-c \gamma \tau}$, from which the dephasing time $T_{2}=1 / \gamma$ can be determined if the coefficient $c$ is known. ${ }^{3}$ For a purely homogeneously (inhomogeneously) broadened two-level system, $c=2$ $(c=4)$. This technique has been applied in various bulk semiconductors, such as $\mathrm{CdSe},{ }^{3-5} \mathrm{GaAs},{ }^{6,7} \mathrm{Ge},{ }^{8} \mathrm{ZnSe},{ }^{9}$ or $\mathrm{GaSe},{ }^{10,11}$ or quantum wells manufactured in binary materials like GaAs (Ref. 12) and ternary materials like $\mathrm{In}_{x} \mathrm{Ga}_{1-x}$ As. $^{13}$ These studies have demonstrated that inhomogeneous broadening is present in many solid-state materials. The inhomogeneous broadening can be due to environmental resonance shifts caused by, e.g., crystal fields or strain as well as interface disorder ${ }^{14}$ in the quantum-well structures. Compared to the homogeneous linewidth, reflecting the phase distorting (phonon or carrier-carrier) scattering processes, the inhomogeneous linewidth is often much broader. Within the inhomogeneous line, the homogeneous linewidth can be determined by spectral hole burning experiments, ${ }^{15}$ or photon-echo experiments ${ }^{16,17}$ in the time domain. In case the inhomogeneous linewidth is comparable to or smaller than the homogeneous linewidth, it is normally difficult to get accurate information on the two broadening mechanisms.
If a nearly degenerate three-level system is coherently excited by an ultrashort laser pulse, the correlation trace in a TFWM experiment may additionally show modulations due to quantum interferences or beats

$$
I_{\mathrm{TFWM}}(\tau)=I_{\mathrm{ave}}(\tau)\left\{1+I_{m}(\tau) \cos (\Delta \omega \tau+\phi)\right\},
$$

where $\Delta \omega=\Delta E / \hbar$ is determined by the splitting $\Delta E=E_{2}-E_{1}$ of the nearly degenerate transition energies $E_{1}$ and $E_{2}$. The decays of the average signal $I_{\text {ave }}(\tau)$ and of the modulation amplitude $I_{m}(\tau)$ are again determined by the dephasing times of the transitions involved. The phase of the modulation is specified by $\phi$. Such modulations have been observed between various excitonic resonances in GaAs quantum wells ${ }^{18-20}$ as well as in bulk CdSe. $^{4,5}$

Recently, it was shown that spectral resolution of the FWM signal provides the possibility to distinguish between polarization interference from independent macroscopic polarizations and true quantum interferences in material systems, where more transitions share a common level. ${ }^{21}$ The theoretical basis for this work is given by the optical-Bloch equations OBE's. In our model the OBE's are solved, in the time domain, for either independent two-level systems and for three-level systems. By Fourier transforming this result, we get the spectral behavior, which we investigate in the experiments. ${ }^{22}$

In this work, we study the influence of inhomogeneous broadening on the TFWM signal. With spectral resolution it is possible to deduce the homogeneous linewidth $\gamma$ and the inhomogeneous linewidth $\Gamma$ simultaneously. When the interfering resonances share a common level, it is necessary, in addition to the inhomogeneous broadening, to describe how the inhomogeneous broadenings of the different resonances are correlated. ${ }^{23}$ In an atomicgas system, which normally is Doppler broadened, one expects a priori full correlation, whereas this condition could be relaxed in a condensed matter system.

Experimentally, we discuss results obtained by spectrally resolved TFWM for the impurity-bound excitons $\left(I_{1}\right.$ and $\left.I_{2}\right)$ in CdSe and the heavy-hole exciton (hhx) and 
light-hole exciton (lhx) in a GaAs multiple quantum well (MQW). The present work is of special importance for artificially grown semiconductor structures because the optical resonances in these, in many cases, are inhomogeneously broadened due to unavoidable interface roughness.

\section{LINE-SHAPE ANALYSIS FOR SPECTRALLY RESOLVED TFWM}

The theoretical basis for spectrally resolved four-wave mixing has been developed in a recent paper. ${ }^{22}$ In this section, we discuss the extension of the theory to inhomogeneously broadened systems. We have calculated the third-order polarization $P^{(3)}(t, \tau)$ from the optical-Bloch equations. In the calculation, we only keep the terms having the wave vector $2 \mathbf{k}_{2}-\mathbf{k}_{1}$, since we experimentally detect in this direction. Contributions to the TFWM signal resulting from pulse overlap in the sample are neglected in the present calculations. The TFWM signal, which we detect with a spectrometer is $I_{\text {TFWM }} \propto\left[P^{(3)}(\tau, \omega)+\text { c.c. }\right]^{2}$, where $P^{(3)}(\tau, \omega)$ is the Fourier transform with respect to $t$ of $P^{(3)}(\tau, t)$. We make use of the complex frequencies $\Omega_{i j}=\omega_{i j}-i \gamma_{i j}$, where $\gamma$ is the damping. In order to develop some intuition, we first consider the response from one homogeneously broadened resonance. The result is ${ }^{22}$

$P_{\text {hom }}^{(3)}(\tau, t) \propto 2 N M_{21}^{4} e^{-i \Omega_{21}(t-\tau)} e^{i \Omega_{21}^{*} \tau} \Theta(t-\tau) \Theta(\tau)$,

where $M$ is the dipole matrix element and $t$ is the time after the arrival of the first pulse and the second pulse arrives at $t=\tau$. The spectral overlap of the resonance with the incident fields as well as various integrations involving the electric fields of the incident pulses have been suppressed. $N$ denotes the number of two-level "atoms" and $\Theta(t)$ is the Heaviside step function. Fourier trans- forming Eq. (2), one gets

$$
P_{\text {hom }}^{(3)}(\tau, \omega) \propto N \frac{2 M_{21}^{4} e^{i \Omega_{21}^{*} \tau}}{\Omega_{21}-\omega} \Theta(\tau) .
$$

It can immediately be seen that $I_{\mathrm{TFWM}}(\tau)$ $\propto \exp \left(-2 \tau / T_{2}\right)$, and that the spectrum of $I_{\mathrm{TFWM}}$ is a Lorentzian.

If the resonance is inhomogeneously broadened, the result in Eq. (2) must be integrated over, e.g., a Gaussian distribution of transition frequencies

$g_{N}\left(\omega_{21}\right)=\frac{\sqrt{4 \ln 2}}{\Gamma \sqrt{\pi}} \exp \left[-4 \ln 2\left[\frac{\omega_{21}^{c}-\omega_{21}}{\Gamma}\right]^{2}\right]$,

where the inhomogeneous broadening has a full width at half maximum (FWHM) of $\Gamma$. The polarization in real time is now found from

$$
\begin{aligned}
P_{\mathrm{inh}}^{(3)}(\tau, t) & \propto \int g_{N}\left(\omega_{21}\right) P_{\mathrm{hom}}^{(3)}\left(\tau, t, \omega_{21}\right) d \omega_{21} \\
& =P_{\mathrm{hom}}^{(3)}\left(\tau, t, \omega_{21}^{c}\right) \exp \left[-\frac{\Gamma^{2}(t-2 \tau)^{2}}{16 \ln 2}\right] .
\end{aligned}
$$

The result obtained in Eq. (5) shows that the photon echo emitted at $t=2 \tau$ is the real-time Fourier transform of the inhomogeneous line. The $\Theta$ function clips the Gaussian in real time for delays smaller than the inverse linewidth $\tau<1 / \Gamma$. Figure 1 shows the normalized absolute square of Eq. (5) as a function of real time $t$ for different delays. Figure 1 illustrates that the photon echo comes out without being clipped only after a certain delay as explained above. For delays shorter than $1 / \Gamma$, the response will have a broader frequency content due to the $\theta$ function, which, in the experiments, has a rise time given by the length of the laser pulse. Fourier transforming Eq. (5) gives

$$
\begin{aligned}
P_{\mathrm{inh}}^{(3)}(\tau, \omega) \propto 4 i N M_{21}^{4} \frac{\sqrt{\pi \ln 2}}{\Gamma} e^{i 2 \omega \tau} e^{-2 \gamma_{21} \tau} \exp \left[-4 \ln 2 \frac{\left(\omega_{21}^{c}-\omega\right)^{2}-\gamma_{21}^{2}}{\Gamma^{2}}\right) \\
\quad \times \exp \left[i 8 \ln 2 \frac{\left(\omega_{21}^{c}-\omega\right) \gamma_{21}}{\Gamma^{2}}\right) \operatorname{erfc}\left[2 \sqrt{\ln 2}\left[\frac{\gamma_{21}}{\Gamma}-\frac{\Gamma_{\tau}}{8 \ln 2}+i \frac{\omega_{21}^{c}-\omega}{\Gamma}\right]\right]
\end{aligned}
$$

In this case, $I_{\mathrm{TFWM}}(\tau) \propto \exp \left(-4 \tau / T_{2}\right)$ for $\tau>5 / \Gamma .^{24}$ The resonance enhancement is now a Gaussian, which also reflects the spectrum of $I_{\text {TWFM }}$ at sufficiently large delay $(\tau \gg>1 / \Gamma)$, when the erfc becomes independent of the delay. This is illustrated in Fig. 2, where the absolute square of Eq. (6) is shown versus delay and for different detunings $\delta=\left(\omega-\omega_{21}^{c}\right) / \Gamma$. In Fig. 2(a), for a small inhomogeneous broadening $(\Gamma=3 \gamma)$, there is a small dependence on detuning, reflecting that the spectrum becomes narrower for increasing delay. This is due to the discontinuity in the real-time behavior for delays shorter than the inverse inhomogeneous broadening, as shown in Fig. 1. This is expressed in Eq. (6) in the erfc function. For a larger inhomogeneous broadening, as in Fig. 2(b) with $\Gamma=10 \gamma$, the dependence on detuning is much stronger and, for small detunings, the correlation traces even show a distinct maximum. This behavior reflects how inhomogeneous broadening and the resulting photon echo is revealed in spectrally resolved four-wave mixing, allowing a simultaneous determination of the homogeneous linewidth and the inhomogeneous linewidth. The expression in Eq. (6) also contains a phase factor, where the magnitude of the phase shift through the resonance is determined by the ratio $\beta=\Gamma / \gamma$. This is the equivalent of the phase shift introduced by the resonance denominator in the case of homogeneous broadening in Eq. (3). 


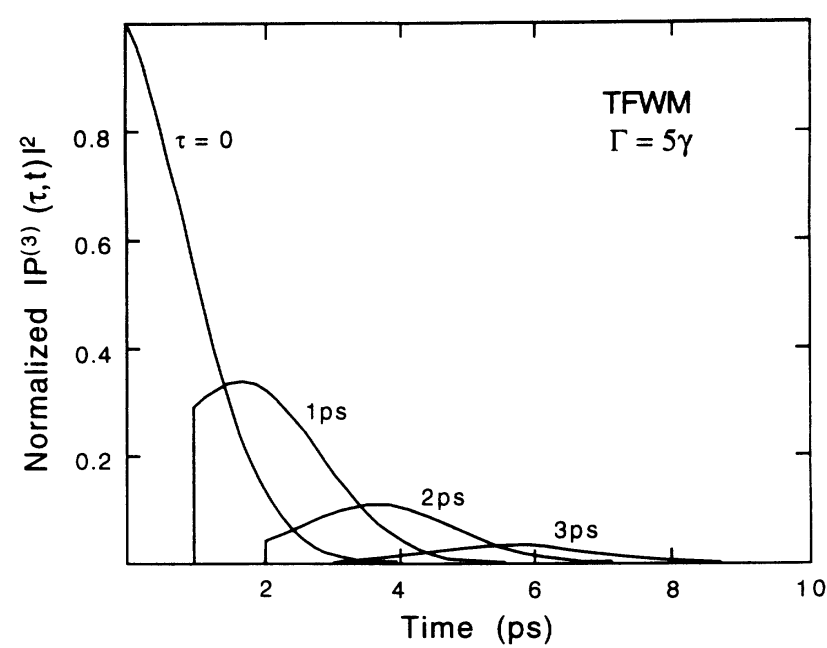

FIG. 1. The normalized absolute square of Eq. (5) vs real time $t$ for different delays $\tau=0,1,2$, and 3 ps. For this calculation $\Gamma=5 \gamma$.

Recently, such Lorentz-Gauss (LG) profiles were studied in detail in GaAs MQW by Humliček et al. ${ }^{25}$ They showed that the LG profiles are Lorentzian in the wings and Gaussian in the center of the line. They found indeed, that the absorption profiles in GaAs MQW are well described by a LG profile at not too low temperatures.

If several such independent resonances are coherently excited by the same laser pulse, the total polarization is calculated as a sum of terms like Eq. (6), for positive de-

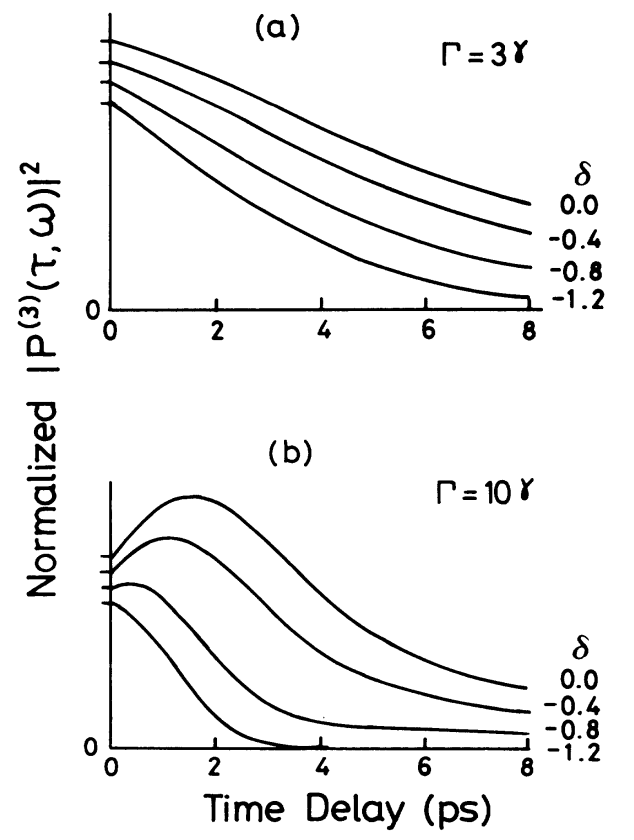

FIG. 2. The normalized absolute square of Eq. (6) showing the calculated decay of the TFWM signal vs delay $\tau$ for different detunings $\delta=\left(\omega-\omega_{\text {res }}\right) / \Gamma$. For clarity the curves are offset by 0.1 . In (a), $\Gamma=3 \gamma$ and in (b), $\Gamma=10 \gamma$. lay $\tau>0$,

$$
P_{\mathrm{PI}}^{(3)}(\tau, \omega) \propto \sum_{i} P_{\mathrm{inh}, i}^{(3)}\left(\tau, \omega, \omega_{21, \mathrm{i}}\right) .
$$

The subscript PI indicates that Eq. (7) results in polarization interference, when the total emitted field from $P_{\mathrm{PI}}^{(3)}(\tau, \omega)$ is squared in the external detector. Note that in the case of inhomogeneous broadening, it is the phase of the Erfc that determines the beat period [see Eq. (6)]. The signature of polarization interference is a phase change of the beats around a resonance, because the beating is between a resonant term and a nonresonant term. The Erfe depends strongly on the ratio $\beta$, which sets the limits for the possibility of observing polarization interference. The emitting polarizations do not interfere for a delay exceeding the inverse inhomogeneous broadening. This behavior can be seen in the calculation in Fig. 3, where the modulation persists longer for small inhomogeneous broadening [Fig. 3(a)] than for a larger inhomogeneous broadening [Fig. 3(b)]. A small phase shift of the modulation as a function of detuning can be observed, and this again depends on $\beta$, as seen in Eq. (6). For small inhomogeneous broadening, it is clear that another signature of polarization interference is a minimum modulation amplitude at the resonance frequency, $\delta=0$.

Turning to the case where the beating resonances are coupled, i.e., forming a three-level system, one must reconsider the OBE. This has been done previously in a recent publication. ${ }^{22}$ The result for the third-order polarization $P^{(3)}(\tau, t)$, calculated for positive delay $\tau>0$, is

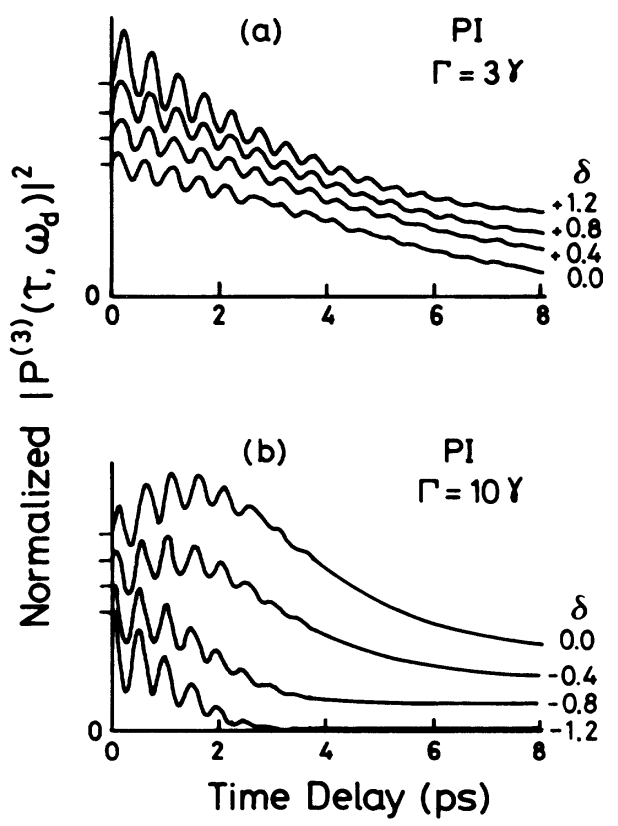

FIG. 3. Polarization interference for different detunings $\delta=\left(\omega-\omega_{21}\right) / \Gamma$ calculated from Eq. (7) for $\hbar \omega_{21}=1.5500 \mathrm{eV}$ and $\hbar \omega_{31}=1.5583 \mathrm{eV}$ corresponding to a beat period of $T_{\text {beat }}=0.5$ ps. $\gamma_{21}=(8 \mathrm{ps})^{-1}, \gamma_{31}=(4 \mathrm{ps})^{-1}, \Gamma_{21}=\Gamma_{31}=\Gamma$, (a) $\Gamma=3 \gamma_{21}$ and (b) $\Gamma=10 \gamma_{21}$. For clarity the curves are offset by 0.2 . 


$$
\begin{aligned}
P_{\mathrm{QB}, \text { hom }}^{(3)}(\tau, t)= & i N M_{21}^{2}\left\{2 M_{21}^{2} e^{-i \Omega_{21}(t-\tau)} e^{i \Omega_{21}^{*} \tau}+M_{31}^{2} e^{-i \Omega_{21}(t-\tau)} e^{i \Omega_{31}^{*} \tau}\right\} \theta(t-\tau) \\
& +i N M_{31}^{2}\left\{2 M_{31}^{2} e^{-i \Omega_{31}(t-\tau)} e^{i \Omega_{31}^{*} \tau}+M_{21}^{2} e^{-i \Omega_{31}(t-\tau)} e^{i \Omega_{21}^{*} \tau}\right\} \theta(t-\tau)
\end{aligned}
$$

where $N$ now is the density of three-level systems. The subscript QB indicates that a true quantum beat is found in a three-level system, because one resonant term contains the phase (in the delay domain) of both resonances. Fourier transforming Eq. (8), we get the result ${ }^{22}$

$$
\begin{aligned}
P_{\mathrm{QB}, \text { hom }}^{(3)}(\tau, \omega) \propto N & \frac{2 M_{21}^{4} e^{i \Omega_{21}^{*} \tau}+M_{21}^{2} M_{31}^{2} e^{i \Omega_{31}^{*} \tau}}{\Omega_{21}-\omega} \\
& \left.+\frac{2 M_{31}^{4} e^{i \Omega_{31} \tau}+M_{31}^{2} M_{21}^{2} e^{i \Omega_{21}^{*} \tau}}{\Omega_{31}-\omega}\right\} .
\end{aligned}
$$

Assuming equal dipole matrix elements of the transitions and distinctly different dampings of the two levels involved in the beating, e.g., $\gamma_{31} \gg \gamma_{21}$, a simple expression [compare with Eq. (1)] for the intensity of the TFWM signal detected near one resonance is obtained

$I_{\mathrm{TFWM}} \propto \frac{e^{-2 \gamma_{21} \tau}\left\{1+e^{-\gamma_{31} \tau} \cos \left[\left(\omega_{21}-\omega_{31}\right) \tau\right]\right\}}{\left(\omega_{21}-\omega\right)^{2}+\gamma_{12}^{2}}$,

showing that the average signal decays with the slowest damping rate, whereas the decay of the beat modulation decays with the fastest damping rate.

We introduce a correlated distribution of $\omega_{21}$ and $\omega_{31}$ to describe the inhomogeneous broadening in a threelevel system. If there is no correlation, $\delta \omega_{2}$ is independent of $\delta \omega_{1}$. On the other hand, full correlation describes the situation where $\delta \omega_{2}$ is a function of $\delta \omega_{1}$, $\delta \omega_{2}=f\left(\delta \omega_{1}\right)$. The two-dimensional Gaussian is ${ }^{23}$

$g_{N}\left(\omega_{21}, \omega_{31}, \lambda\right)=\frac{4 \ln 2 \sqrt{1-\lambda^{2}}}{\pi \widetilde{\Gamma}_{21} \widetilde{\Gamma}_{31}} \exp \left\{-4 \ln 2\left[\left(\frac{\omega_{21}-\omega_{21}^{c}}{\widetilde{\Gamma}_{21}}\right]^{2}-2 \lambda \frac{\left(\omega_{21}-\omega_{21}^{c}\right)\left(\omega_{31}-\omega_{31}^{c}\right)}{\widetilde{\Gamma}_{21} \widetilde{\Gamma}_{31}}+\left(\frac{\omega_{31}-\omega_{31}^{c}}{\widetilde{\Gamma}_{31}}\right)^{2}\right]\right\}$,

where $\lambda$ is the correlation parameter and $\omega_{i 1}^{c}$ is the center frequency of the inhomogeneously broadened line. $\lambda=0$ $(\lambda=1)$ means no (full) correlation. In Eq. (11), $\widetilde{\Gamma}_{i 1}=\Gamma_{i 1}\left(1-\lambda^{2}\right)^{1 / 2}$. Notice that the correlation makes the effective linewidth smaller, when $\lambda$ increases from 0 towards 1 .

Calculating the polarization from an inhomogeneously broadened three-level system, we notice that the first term in Eq. (8) is similar to the result for a single resonance. Therefore, we focus on the second term from Eq. (8), which we denote the beating term

$$
P_{\text {beat,hom }}^{(3)}(\tau, t)=i N M_{21}^{2} M_{31}^{2} e^{-i \Omega_{21}(\tau-t)} e^{i \Omega_{31}^{*} \tau}
$$

and we consider only the contribution from one resonance, that is $\omega \approx \omega_{21}$. From Eq. (11) and Eq. (12) we get

$$
\begin{aligned}
& P_{\text {beat, inh }}^{(3)}(\tau, t)=\iint d \omega_{21} d \omega_{31} g_{N}\left(\omega_{21}, \omega_{31}, \lambda\right) P_{\text {beat, hom }}^{(3)}\left(\tau, t, \omega_{21}, \omega_{31}\right) \\
& =i N M_{21}^{2} M_{31}^{2} e^{-i \omega_{21}^{c}(t-\tau)} e^{-\gamma_{21}(t-\tau)} e^{-\gamma_{31}{ }^{\tau}} e^{i \omega_{31}^{c} \tau} \exp \left(-\frac{\Gamma_{21}^{2}(t-\tau)^{2}}{16 \ln 2}\right) \\
& \times \exp \left(-\frac{\Gamma_{31}^{2} \tau^{2}}{16 \ln 2}\right) \exp \left(\lambda \frac{\Gamma_{21} \Gamma_{31}(t-\tau) \tau}{8 \ln 2}\right) \theta(t-\tau)
\end{aligned}
$$

i.e., the beating term gives rise to a signal in real time emitted at $t=\tau$. Notice that it is the real inhomogeneous broadening, $\Gamma_{i 1}$, which enters in Eq. (13). This signal from Eq. (13) will, in addition to the $T_{2}$ damping, be damped by a Gaussian with a fixed FWHM related to the inhomogeneous broadening of one resonance in case of no correlation $(\lambda=0)$ or with a $t$-dependent and $\tau$-dependent FWHM related to both inhomogeneous broadenings in case of correlation $(\lambda \neq 0)$. In Eq. (13), the phase of both resonances appear. Fourier transforming Eq. (13), we obtain the result for the beating term 


$$
\begin{aligned}
& \left.P_{\text {beat, inh }}^{(3)}(\tau, \omega)=i N M_{21}^{2} M_{31}^{2} 2 \frac{\sqrt{\pi \ln 2}}{\Gamma_{21}} \exp \left\{i\left(\omega_{31}^{c}+\omega\right) \tau\right\} \exp \left\{-\mid \gamma_{31}+\lambda \gamma_{21} \frac{\Gamma_{31}}{\Gamma_{21}}\right) \tau\right\} \exp \left\{-\left(1-\lambda^{2}\right) \frac{\Gamma_{31}^{2} \tau^{2}}{16 \ln 2}\right\} \\
& \times \exp \left\{-4 \ln 2 \frac{\left(\omega_{21}^{c}-\omega\right)^{2}-\gamma_{21}^{2}}{\Gamma_{21}^{2}}\right\} \exp \left\{i \frac{\omega_{21}^{c}-\omega}{\Gamma_{21}}\left[\frac{8 \ln 2 \gamma_{21}}{\Gamma_{21}}-\lambda \Gamma_{31} \tau\right]\right\} \\
& \times \operatorname{erfc}\left\{2 \sqrt{\ln 2}\left(\frac{i\left(\omega_{21}^{c}-\omega\right)+\gamma_{21}}{\Gamma_{21}}-\lambda \frac{\Gamma_{31} \tau}{8 \ln 2}\right)\right\} .
\end{aligned}
$$

In Eq. (14), the effect of the correlation becomes evident. The beating term will decay both from a $T_{2}$ term and a Gaussian term in case of no correlation. For large correlation the Gaussian decays very slowly, and therefore in a quantum-beat experiment, the modulation persists as long as the signal does. The two first resonant terms $\left(\omega \approx \omega_{21}\right)$ from Eq. (8) then give the polarization

$$
\begin{aligned}
P_{\mathrm{QB}, \text { inh }}^{(3)}(\tau, \omega)= & 2 i N M_{21}^{2} \frac{\sqrt{\pi \ln 2}}{\Gamma_{21}} \exp \{i \omega \tau\} \exp \left\{i \frac{\left(\omega_{21}^{c}-\omega\right)}{\Gamma_{21}} \frac{8 \ln 2 \gamma_{21}}{\Gamma_{21}}\right\} \exp \left\{-4 \ln 2 \frac{\left(\omega_{21}^{c}-\omega\right)^{2}-\gamma_{21}^{2}}{\Gamma_{21}^{2}}\right\} \\
\times & 2 M_{21}^{2} \exp \left\{i \omega \tau-2 \gamma_{21} \tau\right) \operatorname{erfc}\left\{2 \sqrt{\ln 2}\left[\frac{i\left(\omega_{21}^{c}-\omega\right)+\gamma_{21}}{\Gamma_{21}}-\frac{\Gamma_{21} \tau}{8 \ln 2}\right]\right\} \\
& +M_{31}^{2} \exp \left\{i \omega_{31}^{c} \tau-\left\{\gamma_{31}+\lambda \gamma_{21} \frac{\Gamma_{31}}{\Gamma_{21}}\right] \tau\right\} \exp \left\{-i \lambda \frac{\omega_{21}^{c}-\omega}{\Gamma_{21}} \Gamma_{31} \tau\right\} \\
& \left.\times \exp \left\{-\left(1-\lambda^{2}\right) \frac{\Gamma_{31}^{2} \tau^{2}}{16 \ln 2}\right\} \operatorname{erfc}\left\{2 \sqrt{\ln 2}\left\{\frac{i\left(\omega_{21}^{c}-\omega\right)+\gamma_{21}}{\Gamma_{21}}-\lambda \frac{\Gamma_{31} \tau}{8 \ln 2}\right]\right\}\right] .
\end{aligned}
$$

If the two beating resonances are separated much more than their respective inhomogeneous broadenings, the main contribution to the detected signal near one resonance is given by Eq. (15). If not, one needs to add a contribution that can be obtained from Eq. (15) with exchange of indexes $(2 \Longleftarrow 3)$. In the following, we focus on the former case. Figure 4 shows the calculated depen- dence on detuning of the TFWM signal for small inhomogeneous broadening $(\Gamma=3 \gamma)$. In contrast to polarization interference, there is no phase shift at the resonance and the modulation has maximum amplitude in the resonance. Therefore, it is possible to distinguish between polarization interference (independent two-level systems) and quantum beats (three-level systems). ${ }^{21}$ Here again,

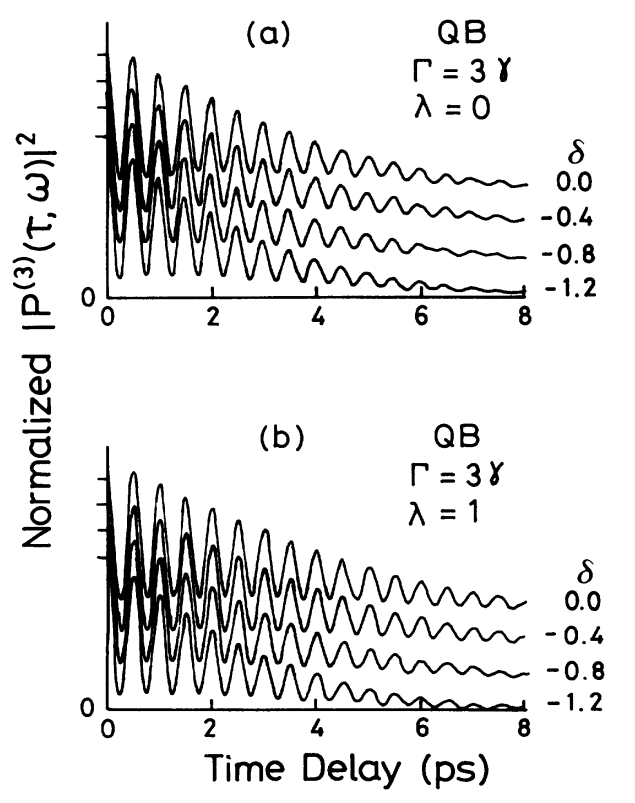

FIG. 4. Quantum beats calculated from Eq. (15) for parameters as in Fig. 3 and $\Gamma=3 \gamma$ (a) zero correlation $\lambda=0$ and (b) full correlation $\lambda=1$.

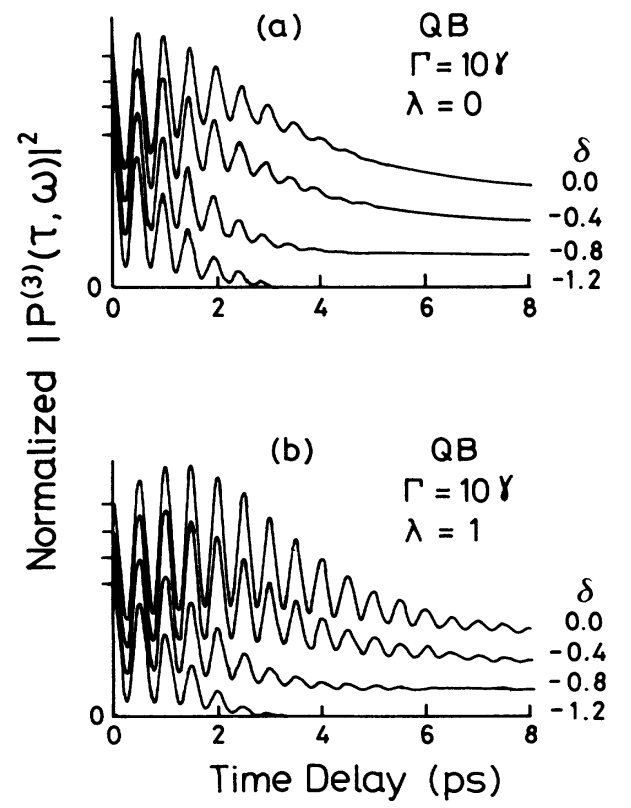

FIG. 5. Quantum beats calculated from Eq. (15) for parameters as in Fig. 3 and $\Gamma=10 \gamma$ (a) zero correlation $\lambda=0$ and (b) full correlation $\lambda=1$. 
the influence of inhomogeneous broadening is dependent on detuning and time delay: For large detuning, the correlation traces decay faster. In Fig. 4(a), we have chosen $\lambda=0$, and the beats disappear before the signal does. In the other extreme, $\lambda=1$, the beats persist as long as the signal does. In a quantum-beat experiment, it is, therefore, possible to deduce how strong the beating resonances are correlated. If the resonances are more inhomogeneously broadened, the signal, as a function of delay, again shows a maximum for a finite delay. However, the maximum is less distinct for smaller correlation. This can be observed in Fig. 5(a) with zero correlation $\lambda=0$ and in Fig. 5(b) with full correlation $\lambda=1$, respectively.

After a short description of our experimental setup, we shall present our experimental results demonstrating the influence of inhomogeneous broadening on spectrally resolved four-wave mixing.

\section{EXPERIMENTAL DETAILS}

We have investigated spectrally resolved TFWM spectra from two different material systems: (a) a thin $(5 \mu \mathrm{m})$ bulk CdSe sample and (b) two molecular-beam epitaxially grown $\mathrm{GaAs} / \mathrm{Al}_{x} \mathrm{Ga}_{1-x} \mathrm{As}(x \approx 0.3) \mathrm{MQW}$ samples with well width of 80 and $100 \AA$, respectively. The CdSe sample is of high optical quality with pronounced $I_{1}$ (exciton bound to neutral acceptor) and $I_{2}$ (exciton bound to neutral donor) lines. The energies are $E_{I 1}=1.8169 \mathrm{eV}$ and $E_{I 2}=1.8217 \mathrm{eV}$. The GaAs MQW samples are grown on (100)-oriented GaAs substrates with 20 (10) wells of width of $100 \AA$ ( $80 \AA$ ) corresponding to 35 (28) monolayers. The 100- $\AA$ GaAs MQW sample has a linewidth (FWHM) of the hhx (absorption peak at $E_{\mathrm{hhx}}=1.5500$ $\mathrm{eV}$ ) of $\Gamma_{\mathrm{hhx}}=1.4 \mathrm{meV}$ and the lhx (absorption peak at $E_{\mathrm{lhx}}=1.5580 \mathrm{eV}$ ) is approx 1.4 times broader. The observed Stokes shift of the luminescence was $0.5 \mathrm{meV}$. For the $80-\AA$ GaAs MQW sample, we have a linewidth (FWHM) of the hhx (absorption peak at $E_{\mathrm{hhx}}=1.5672$ $\mathrm{eV}$ ) of $\Gamma_{\mathrm{hhx}}=1.8 \mathrm{meV}$, with a Stokes shift of $0.5 \mathrm{meV}$. With this sample, it was possible to excite the hhx without the lhx (absorption peak at $E_{\mathrm{lhx}}=1.5805 \mathrm{eV}$ ), whereby the beats were avoided. The molecular-beamepitaxy samples appeared to be spatially inhomogeneous with varying linewidth, when the laser was moved on the sample. The samples were mounted in a liquid helium cryostat, and the presented spectra are obtained at low temperatures between $T=2 \mathrm{~K}$ and $T=10 \mathrm{~K}$. For the CdSe experiments, we used pulses generated at a repetition rate of $82 \mathrm{MHz}$ from a mode-locked argon-ion laser, which pumps synchronously a tunable dye laser (DCM). The generated pulses have a spectral width of about 4 meV corresponding to a coherence time of approx $500 \mathrm{fs}$. The linearly copolarized light is incident on the crystal with the polarization perpendicular to the $c$ axis $\left(E_{\perp} c\right)$. In the GaAs MQW experiments, we used pulses generated at a repetition rate of $80 \mathrm{MHz}$ from a self-mode-locked Ti:Sapphire laser. The pulse length was approx $150 \mathrm{fs}$ corresponding to a spectral width of about $11 \mathrm{meV}$. The laser spot on the sample is focused to approx. $40 \mu \mathrm{m}$ and the self-diffracted TFWM signal from the sample is dispersed in a spectrometer with a resolution of less than $0.1 \mathrm{meV}$ and detected time integrated.

\section{EXPERIMENTAL RESULTS AND DISCUSSION}

In CdSe we have measured the spectrally resolved decay from an impurity-bound exciton, the $I_{1}$ line as shown in Fig. 6. The peak observed in Fig. 6 at zero delay, $\tau=0$, is due to pulse overlap in the sample. In the calculations we have not included terms due to pulse overlap and, therefore, we do not consider this part of the experiment. The observed spectral dependence (full curves) in Fig. 6 is seen to be in agreement with Fig. 3, as the more detuned spectral components decay faster than the center component. This is the signature of the emission of a photon echo, when the signal is spectrally resolved rather than real-time resolved. The information in the measurements is the same, although it is easier to see the presence of a photon echo in real time. A fit (dashed curves in Fig. 6) to the $\delta=0$ component gave the parameters $T_{2}\left(I_{1}\right)=200 \mathrm{ps}$ and $\Gamma\left(I_{1}\right)=50 \gamma\left(I_{1}\right)=0.16 \mathrm{meV}$ in reasonable agreement with the observed linewidth in a linear luminescence experiment. These values are also in agreement with recent real-time resolved photon-echo measurements in CdSe by Schwab, Lyssenko, and Hvam. ${ }^{17}$ The two detuned spectral components in Fig. 6 are not fitted separately, but calculated with the same parameters as for the $\delta=0$ component. The calculated curves agree excellently with the observations.

In a recent paper, ${ }^{21}$ we studied the nature of four-wave mixing beats in semiconductors. We showed results from a polarization interference experiment exciting simultaneously the $I_{1}$ and $I_{2}$ bound-exciton lines in CdSe. The experiments gave the expected phase shift of the beats in the resonance (see Ref. 21 for more details).

Performing a similar experiment in the $80-\AA$ GaAs MQW with the intensity peak of the laser tuned well below the hhx resonance, we could avoid the beats with

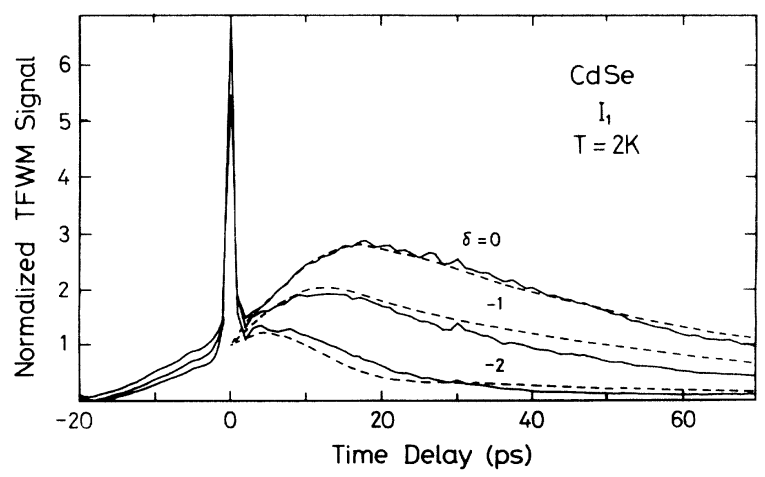

FIG. 6. Spectrally resolved TFWM (full curves) at the impurity-bound exciton $I_{1}$ in CdSe at $T=2 \mathrm{~K}$ with resonant laser excitation. The detuning is $\delta=\hbar\left(\omega-\omega_{I 1}\right) /(0.06 \mathrm{meV})$. Each curve is normalized at zero delay $(\tau=0)$ suppressing the peak. The dashed curves are calculations from Eq. (6) with the parameters $T_{2}\left(I_{1}\right)=200 \mathrm{ps}$ and $\Gamma=50 \gamma$ (see text). 


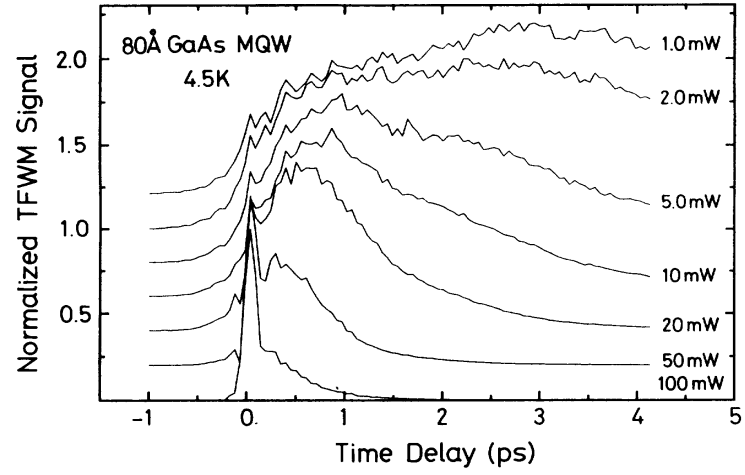

FIG. 7. Spectrally resolved TFWM in a $80-\AA$ GaAs MQW sample for different average power per beam (see text). Only the resonant trace for the $\mathrm{hhx}\left(\omega=\omega_{\mathrm{hhx}}\right)$ is shown for each intensity. The curves are offset by 0.2 , and each curve is normalized at $\tau=0$.

the lhx. At the temperature of $T=4.5 \mathrm{~K}$, we recorded the signal as a function of average power per beam from $I_{\text {ave }}=1 \mathrm{~mW}$ (average exciton density per layer $3 \times 10^{9}$ $\mathrm{cm}^{-2}$ ) to $I_{\text {ave }}=100 \mathrm{~mW}$, as shown in Fig. 7. This experiment demonstrates nicely the changes of the correlation traces as a function of the ratio $\beta$ of inhomogeneous to homogeneous broadening. Increasing the intensity, the dephasing time $T_{2}$ decreases due to exciton-exciton interactions (homogeneous broadening), thereby changing $\beta$. Assuming an intensity-independent inhomogeneous broadening of $\Gamma_{\mathrm{hhx}}=0.33 \mathrm{meV}$, we find a change from $\beta=5.4$ at $I_{\text {ave }}=1 \mathrm{~mW}$ per beam to $\beta=4.2$ at $I_{\text {ave }}=10$ $\mathrm{mW}$ per beam. For average powers above $10 \mathrm{~mW}$, the decay versus time delay is nonexponential, however, preserving the distinct maximum for delays larger than zero. This nonexponential decay is attributed to manybody effects, i.e., phase-space filling and fermion exchange, ${ }^{26}$ making the dephasing faster. The excitation density, where the nonexponential decay sets in is in agreement with the prediction of Abram. ${ }^{27}$

The results from the hhx-lhx quantum beats in the $100-\AA$ GaAs MQW obtained at $10 \mathrm{~K}$ is presented in Fig. 8. The TFWM signal is modulated with $T_{\text {beat }}=460 \mathrm{fs}$ corresponding to a hhx-lhx splitting of $8.9 \mathrm{meV}$ (see inset of Fig. 8). The phase of the beats is constant through the resonances showing the true quantum-beat nature of the modulation. For the hhx resonance, the modulation decays faster than the signal does. In the $\mathrm{lhx}$ resonance, the modulation persists as long as the signal does, because of the shorter dephasing time of the lhx resonance compared to the $h h x$ resonance. In both resonances a clear dependence on detuning $\delta$ is observed, as the signals de-

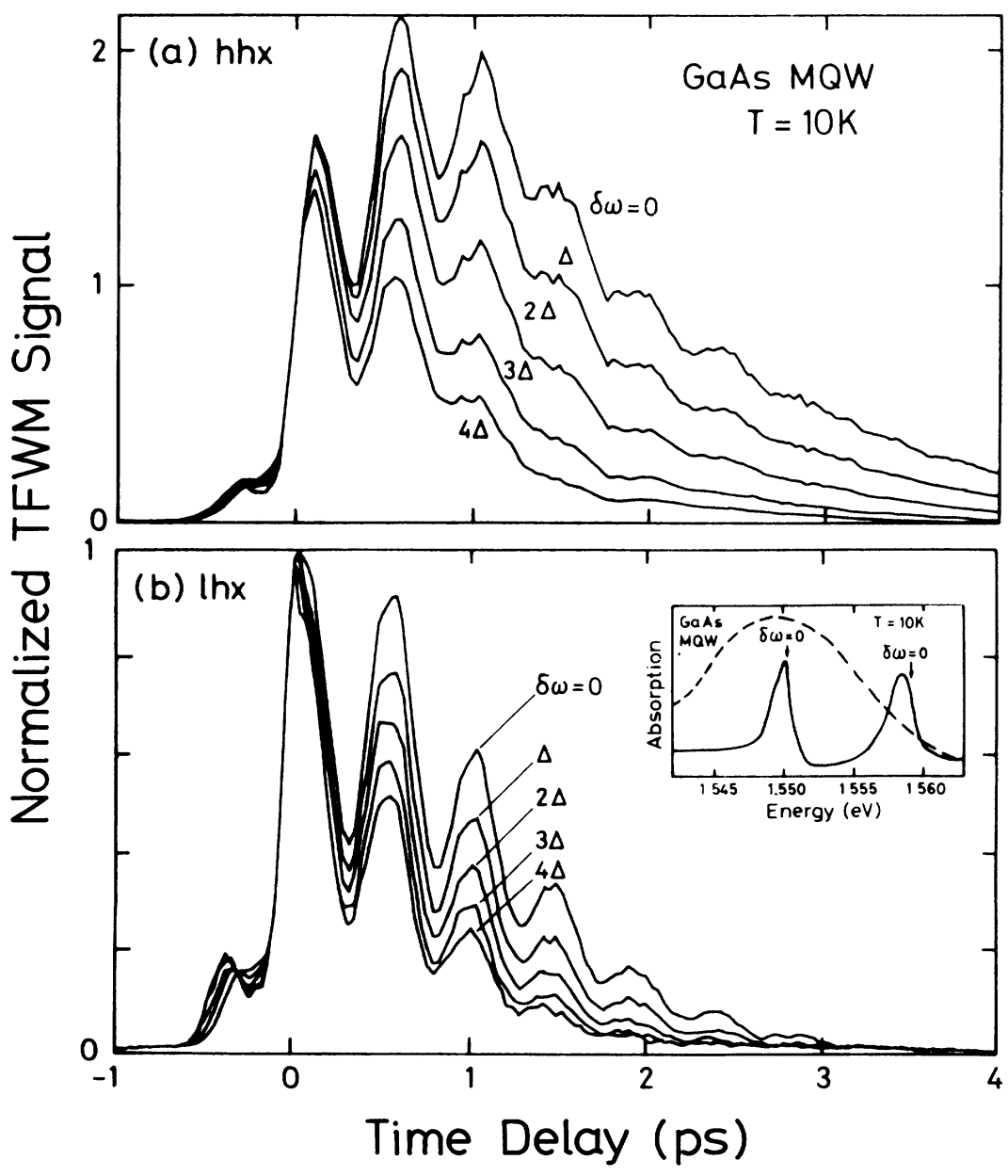

FIG. 8. Spectrally resolved TFWM at hhx (a) and at lhx (b) in a $100-\AA$ MQW. The curves are for different detunings $\delta$, in steps of $\Delta=0.21 \mathrm{meV}$, and all curves are normalized at zero delay, $\tau=0$. The inset shows the absorption spectrum together with the laser profile used in the experiments. In the inset, the arrows indicate zero detuning for the nonlinear resonances. The spectrum was obtained at 10 $\mathrm{K}$ and the average power per beam was 0.8 $\mathrm{mW}$. 


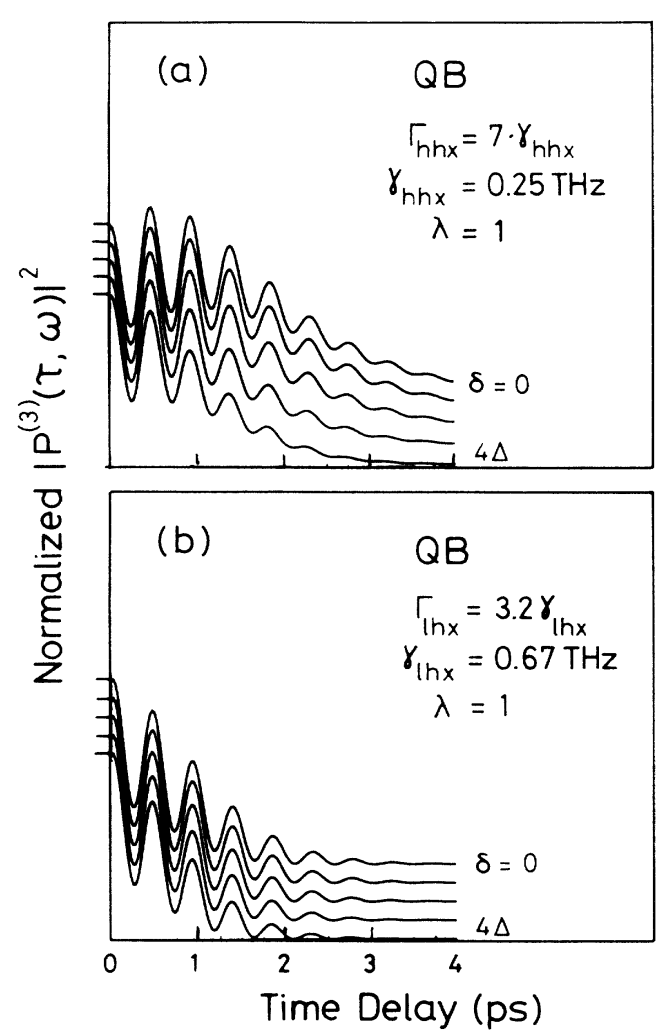

FIG. 9. A calculation of the absolute square of Eq. (15) for parameters in agreement with the observation of Fig. 8. Again, the detunings $\delta$ vary in steps of $\Delta=0.21 \mathrm{meV}$.

cay faster for increasing detuning. This dependence is stronger for the $h \mathrm{hx}$ resonance than for the $\mathrm{hx}$ resonance. From the decay of the lhx signal, where the modulation persists as long as the signal does, we conclude, that the hhx and the lhx quantum beat are highly correlated. In Fig. 9, we show a calculation based on Eq. (15) with parameters relevant for the observations in Fig. 8: $T_{2}(\mathrm{hhx})=4 \mathrm{ps}$ and $T_{2}(\mathrm{hx})=1.5 \mathrm{ps}, \Gamma_{\mathrm{hhx}}=7 \gamma_{\mathrm{hhx}}$ and $\Gamma_{\mathrm{lhx}}=3.2 \gamma_{\mathrm{lhx}}$. The corresponding intensity FWHM linewidth are $\Gamma_{\text {int }}(\mathrm{hhx})=0.8 \mathrm{meV}$ and $\Gamma_{\text {int }}(\mathrm{lhx})=1.0$ $\mathrm{meV}$, which are smaller than the linewidth observed in a linear experiment. However, in the linear experiment a much larger area of the sample was investigated giving the larger inhomogeneous broadening. This shows that it is difficult to compare observations from linear and nonlinear experiments directly. With the present analysis, it is possible to extract the actual homogeneous and inhomogeneous linewidth in a nonlinear experiment.

\section{CONCLUSIONS}

We demonstrate in this paper that extra information is obtained with spectral resolution of the nonlinear signal in a time-integrated transient four-wave mixing experiment, thus representing a useful alternative to real-time resolution of the nonlinear signal. With spectral resolution, it is easy to determine whether a transition is homogeneously or inhomogeneously broadened, and we presented a line-shape analysis to extract both the dephasing time $T_{2}$ and the ratio of inhomogeneous to homogeneous broadening $\beta$. We presented also a rigorous line-shape analysis for the case of inhomogeneously broadened multilevel systems, i.e., independent two-level systems and a three-level system. In the former case, the observed polarization interference will show a phase shift of the beats with respect to the detuning. The phase shift is dependent on $\beta$. The beats will persist only for time delays smaller than the inverse inhomogeneous broadening. A similar result is obtained in a three-level system, if the two inhomogeneous broadenings are uncorrelated. For full correlation, the beats persist as long as the nonlinear signal does. This latter case of quantum beats does not show any phase shift of the beats as a function of the detuning. In CdSe, we determined the (intensity) inhomogeneous and homogeneous broadenings of the $I_{1}$ line. In GaAs multiple quantum wells, we observed (1) the change of homogeneous linewidth with intensity of the heavy-hole exciton line and (2) quantum beats between the heavy-hole and light-hole excitons. In the latter case, we could conclude that the inhomogeneous broadenings were highly correlated.

\section{ACKNOWLEDGMENTS}

The authors thank I. Balslev for many enlightening discussions concerning the theory and B.S. Razbirin and D. Birkedal for fruitful cooperation in the laboratory. This work was supported by the Danish Natural Science Research Council. One of us (V.M.) was supported by the Danish Research Academy.
*Permanent address: Dept. of Solid State Electronics, Vilnius University, Saulétekio Ave. 9, Bldg. III, 2054 Vilnius, Lithuania.

†Permanent address: Institute of Microelectronics Technology and Superpure Materials, Chernogolovka, Moscow District 142432, Russia.

${ }^{1}$ See, e.g., review by K.-H. Pantke and J. M. Hvam, Int. J. Mod. Phys. B 8, 73 (1994); E. O. Göbel, Festkoerperprobleme 30, 269 (1990).

${ }^{2}$ T. Yajima and Y. Taira, J. Phys. Soc. Jpn. 47, 1620 (1979).

${ }^{3}$ C. Dörnfeld and J. M. Hvam, IEEE J. Quantum Electron. QE-25, 904 (1989).

${ }^{4}$ K.-H. Pantke, V. G. Lyssenko, B. S. Razbirin, J. Erland, and J.
M. Hvam, in Proceedings of the 21st International Conference on the Physics of Semiconductors, edited by K. Huang and L. L. Chang (World Scientific, Singapore, 1993), p. 129.

${ }^{5}$ J. Erland, B. S. Razbirin, V. G. Lyssenko, K.-H. Pantke, and J. M. Hvam, J. Cryst. Growth 138, 800 (1994).

${ }^{6}$ L. Schultheis, J. Kuhl, A. Honold, and C. W. Tu, Phys. Rev. Lett. 57, 1635 (1986).

${ }^{7}$ A. Lohner, K. Rick, P. Leisching, A. Leitenstorfer, T. Elsaesser, T. Kuhn, F. Rossi, and W. Stolz, Phys. Rev. Lett. 71, 77 (1993).

${ }^{8}$ T. Rappen, U. Peter, M. Wegener, and W. Schäfer, Phys. Rev. B 48, 4879 (1993).

${ }^{9}$ T. Saiki, K. Takeuchi, K. Ema, M. Kuwata-Gonokami, K. 
Ohkawa, and T. Mitsuyu, J. Cryst. Growth 138, 805 (1994).

${ }^{10} \mathrm{~J}$. M. Hvam and C. Dörnfeld, in Optical Switching in LowDimensional Systems, edited by $\mathbf{H}$. Haug and L. Banyai (Plenum, New York, 1989), p. 233.

${ }^{11}$ F. Minami, A. Hasegawa, T. Kuroda, and K. Inoue, J. Lumin. 53, 371 (1992).

${ }^{12}$ L. Schultheis, A. Honold, J. Kuhl, K. Köhler, and C. W. Tu, Phys. Rev. B 34, 9027 (1986).

${ }^{13}$ T. F. Albrecht, J. H. H. Sandmann, J. Feldmann, W. Stolz, E. O. Göbel, A. Nebel, C. Fallnich, and R. Beigang, Appl. Phys. Lett. 63, 1945 (1993).

${ }^{14}$ C. Weisbuch, R. Dingle, A. C. Gossard, and W. Wiegmann, Solid State Commun. 38, 709 (1981).

${ }^{15}$ Y. R. Shen, The Principles of Nonlinear Optics (Wiley, New York, 1984), Chap. 13.

${ }^{16}$ G. Noll, U. Siegner, S. G. Shevel, and E. O. Göbel, Phys. Rev. Lett. 64, 792 (1991).

${ }^{17}$ H. Schwab, V. G. Lyssenko, and J. M. Hvam, Phys. Rev. B 44, 3999 (1991).

${ }^{18}$ K. Leo, M. Wegener, J. Shah, D. S. Chemla, E. O. Göbel, T.
C. Damen, S. Schmitt-Rink, and W. Schäfer, Phys. Rev. Lett. 65, 1340 (1990).

${ }^{19}$ B. F. Feuerbacher, J. Kuhl, R. Eccleston, and K. Ploog, Solid State Commun. 74, 1279 (1990).

${ }^{20}$ K.-H. Pantke, D. Oberhauser, V. G. Lyssenko, J. M. Hvam, and G. Weimann, Phys. Rev. B 47, 2413 (1993).

${ }^{21}$ V. G. Lyssenko, J. Erland, I. Balslev, K.-H. Pantke, B. S. Razbirin, and J. M. Hvam, Phys. Rev. B 48, 5720 (1993).

22J. Erland and I. Balslev, Phys. Rev. A 48, 1765 (1993).

${ }^{23}$ S. T. Cundiff, Phys. Rev. A 49, 3114 (1994).

${ }^{24}$ Handbook of Mathematical Functions, edited by $\mathbf{M}$. Abramowitz and I. Stegun (Dover, New York, 1972), Chap. 7.

25J. Humlíček, E. Schmidt, L. Bočánek, and R. Švehla, Phys. Rev. B 48, 5241 (1993).

${ }^{26}$ See, e.g., D. S. Chemla, D. A. B. Miller, and S. Schmitt-Rink, in Optical Nonlinearities and Instabilities in Semiconductors, edited by H. Haug (Academic, London, 1988), p. 83.

${ }^{27}$ I. Abram, Phys. Rev. B 40, 5460 (1989). 